\title{
C3-targeted Complement Inhibitor APL-2
}

National Cancer Institute

\section{Source}

National Cancer Institute. C3-targeted Complement Inhibitor APL-2. NCI Thesaurus.

Code C121135.

A pegylated derivative of the cyclic tridecapeptide compstatin and inhibitor of complement component C3 (C3) activation, with potential use as a treatment for various diseases in which excessive complement activation plays a key role, including paroxysmal nocturnal hemog lobinuria (PNH) and age-related macular deg eneration (AMD). Upon administration, C3-targeted complement inhibitor APL-2 selectively binds to C3 and blocks the cleavage of C3 into C3a and C3b by C3 convertase. This prevents complement pathway activation, and inhibits complement-mediated inflammation and cell lysis. Pegylation increases compstatin's half-life, and increases its efficacy. Excessive complement activation plays a key role in various inflammatory and autoimmune diseases, and leads to tissue destruction. C3 is a crucial and central component of the complement system. 